Journal for

ImmunoTherapy of Cancer

\title{
Strategies for improving the management of immune-related adverse events
}

\author{
Aung Naing (D) , ${ }^{1}$ Joud Hajjar (D) , ${ }^{2}$ James L Gulley (D) , ${ }^{3}$ Michael B Atkins (D) , \\ Gennaro Ciliberto, ${ }^{5}$ Funda Meric-Bernstam, ${ }^{1}$ Patrick Hwu (D) ${ }^{6}$
}

To cite: Naing A, Hajjar J, Gulley JL, et al. Strategies for improving the management of immune-related adverse events. Journal for ImmunoTherapy of Cancer 2020;8:e001754. doi:10.1136/jitc-2020-001754

Accepted 18 November 2020

Check for updates

(C) Author(s) (or their employer(s)) 2020. Re-use permitted under CC BY-NC. No commercial re-use. See rights and permissions. Published by BMJ.

${ }^{1}$ Investigational Cancer Therapeutics, The University of Texas MD Anderson Cancer Center, Houston, Texas, USA

${ }^{2}$ William T Shearer Center for Human Immunobiology, Section of Immunology, Allergy and Retrovirology, Baylor College of Medicine, Houston, Texas, USA

${ }^{3} \mathrm{NCl}$, National Institutes of

Health, Bethesda, Maryland, USA

${ }^{4}$ Oncology, Georgetown

University, Washington, DC, USA

${ }^{5}$ Scientific Directorate, Istituti

Fisioterapici Ospedalieri, Roma, Italy

${ }^{6}$ Division of Cancer Medicine, The University of Texas MD Anderson Cancer Center, Houston, Texas, USA

Correspondence to

Dr Aung Naing;

anaing@mdanderson.org

\section{ABSTRACT}

With the advent of immunotherapeutic agents, durable and dramatic responses have been observed in several hard-to-treat malignancies, outlining a roadmap to conquering cancer. Immune checkpoint inhibitors (ICPi) are a class of immunotherapeutic agents that attack the tumor cells by reinvigorating the suppressed immune system. However, the unbridled T-cell activity disrupts the immune homeostasis and induces a unique spectrum of side effects called immune-related adverse events (irAEs) in a significant proportion of patients. These irAEs are distinct from the side effects produced by traditional chemotherapeutic agents. Although majority of irAEs are manageable with corticosteroids and other immunosuppressive agents, life-threatening and fatal events have been reported. In the absence of predictive biomarkers to identify patients at risk for irAEs and standardized approach to detect, report, and treat irAEs, management of irAEs has been challenging to the patients, caregivers and the healthcare providers alike. With increasing use of ICPis for treatment of various cancers, the incidence of irAEs will undoubtedly increase. There is a compelling need to develop measures to effectively manage irAEs, both in the community settings and in cancer centers alike. To this end, in this paper, we propose several strategies, such as providing patient education, harmonizing irAE management guidelines, standardizing reporting of irAEs, optimizing the choice of immunosuppressive agents, conducting preclinical, clinical and translational studies to better understand irAEs, including high-risk patients, incorporating diagnostic tools to personalize irAE management using wireless technology and digital health, providing a platform to hear the missing patient's voice, and sharing evolving data to improve the management of irAEs.

In recent years, immune checkpoint inhibitors (ICPis) - cytotoxic T lymphocyte antigen 4 (CTLA-4), programmed cell death protein 1 (PD-1), and PD-1 ligand 1 (PD-L1) inhibitors-have revolutionized the treatment of advanced cancer. ${ }^{1}$ In contrast to conventional cancer therapies that target tumors directly, ICPis act by reinvigorating the suppressed immune system to attack tumor cells. However, by disrupting the homeostatic mechanisms that regulate immune cell functions, which keep in check immune responses to "self," ICPis induce a unique spectrum of side effects called immune-related adverse events (irAEs) in a significant proportion of patients. ${ }^{12}$

The irAEs are diverse. The clinical presentation may vary from mild dermatitis to lifethreatening pneumonitis and myocarditis. The incidence of these irAEs is largely dependent on the class of ICPi used. For example, irAEs have been reported in up to $60 \%-70 \%$ of patients treated with ipilimumab (a CTLA-4 inhibitor $)^{1}$ and in $30 \%$ of patients treated with PD-1 inhibitors; the highest incidence has been reported in patients treated with a combination of ipilimumab and nivolumab (a PD-1 inhibitor). Similarly, among organspecific irAEs, colitis is more common in patients treated with ipilimumab, whereas pneumonitis is more common in patients treated with nivolumab or pembrolizumab (another PD-1 inhibitor). ${ }^{13}$ These irAEs are unpredictable and have important clinical implications. Though most irAEs are mild and reversible, allowing continuation of ICPi therapy with close monitoring, some progress rapidly to severe (grade 3 or higher) lifethreatening or fatal events. ${ }^{45}$ The morbidity associated with these severe irAEs can be debilitating, particularly in older patients, ${ }^{6}$ and in many cases is associated with the need to discontinue therapy, which can have unpredictable consequences on the control of the disease. ${ }^{4}$ Severe irAEs often require multidisciplinary management, frequent hospital visits, and prolonged hospitalizations, leading to increased healthcare costs. ${ }^{4}$ Some irAEs may be associated with permanent organ damage, which can have a major impact on quality of life. Importantly, fulminant irAEs may result in treatment-related deaths. ${ }^{8}$

Though durable responses and survival benefit have been achieved with the use of ICPis in cancer treatment, the inherent risk of irAEs presents a challenge to healthcare 
Table 1 Summary of the recommended strategies for effective management of immune-related adverse events (irAEs)

\begin{tabular}{lll}
\hline \multicolumn{1}{c}{ Action items } & Recommended strategies \\
\hline 1 & Providing patient education & $\begin{array}{l}\text { Use drug-specific wallet cards, educational apps, social networks, and support groups to provid } \\
\text { information regarding irAEs and symptom monitoring } \\
\text { Tailor patient education resources to preferences, and, emotional, literacy, and cultural needs of } \\
\text { the patient }\end{array}$ \\
& $\begin{array}{l}\text { Convene an irAE Management Summit } \\
\text { Refining irAE management } \\
\text { guidelines }\end{array}$ & $\begin{array}{l}\text { Develop toxicity-specific management committees to create evidence-based expert consensus } \\
\text { guidelines }\end{array}$
\end{tabular}
guidelines

Include broad perspectives, such as emergency room physicians, anesthesiologists and surgeons, primary care physicians, patient advocates, and nurses, in guideline development/ review

Publish the outcomes of the activities of the proposed summit

Make the summit a regularly planned effort

$\begin{array}{ll}3 & \text { Standardizing reporting of irAEs } \\ 4 & \begin{array}{l}\text { Optimizing the choice of } \\ \text { immunosuppressive agents }\end{array}\end{array}$

Incorporate SITC CTCAE Task Force irAE-specific module into future versions of the CTCAE

Conduct prospective studies to evaluate safety and efficacy of immunosuppressant agents in irAE management and their impact on response to immune checkpoint inhibitor therapy to optimize the choice, dosing, and duration of use of immunosuppressants in management of irAEs

$5 \quad$ Pursuing better understanding of

Conduct more preclinical, clinical, and translational studies to understand the mechanisms underlying the development of irAEs, determine their possible association with treatment outcomes, identify predictors of toxicity, determine the risk for infections and temporal relationship between use of ICPis and onset of infection, and evaluate the role of prophylactic vaccination and antimicrobial therapy

$6 \quad$ Including high-risk patients

Conduct prospective studies to evaluate safety and efficacy of immune checkpoint inhibitors in special populations with history of primary or secondary immune deficiencies, autoimmune diseases, stem cell or solid organ transplantation, HIV, hepatitis B or C, or prior irAEs

Include translational studies to identify immune markers that predict response and risk for irAEs

Discuss the increased risk associated with immune checkpoint blockade with the patient and caregivers prior to initiation of therapy

Optimize the choice, dosing, and duration of immunosuppressants to provide chronic immunosuppression without negating the benefits of immune checkpoint inhibitors

Develop specific guidelines for use of immune checkpoint inhibitors in high-risk patients

Establish a national registry of high-risk patients with cancer treated with ICPis

7 Incorporating diagnostic tools to Identify markers to predict risk for irAEs personalize irAE management

Develop tools to monitor patients for emergence of irAEs

Validate the immune markers and clinical tools in large, prospective studies for reliability and generalizability

$8 \quad$ Using wireless technology and digital health

Efficiently use wireless technology and digital resources such as IO Tox Management app to equip healthcare providers

Use smartphone-based apps to monitor patients for warning symptoms that indicate impending emergence of irAEs

Institute prompt intervention based on collected information

9 Providing a platform to hear the Monitor longitudinal changes in symptoms using a validated symptom assessment tool such as missing patient's voice the MD Anderson Symptom Inventory for early detection of irAEs

10 Sharing evolving data

Disseminate the results of clinical and translational studies to the scientific community in a timely manner

CTCAE, Common Terminology Criteria for Adverse Events; SITC, Society for Immunotherapy of Cancer.

providers. In contrast, some irAEs, such as vitiligo, have been positively associated with response in melanoma patients treated with ICPis. ${ }^{9}$ Thus, immune modulation using ICPis is like a double-edged sword, representing an urgent need to develop strategies that will improve our handling of irAEs. To address this critical need, we recommend the following measures, which are summarized in table 1 .

\section{PROVIDING PATIENT EDUCATION}

Comprehensive patient education is an essential element of toxicity management and high-quality cancer care. Data suggest that enhanced awareness and thoughtful conversations on what to expect during treatment promote coping skills and resiliency in patients, which ultimately lead to treatment adherence and improved health outcomes. ${ }^{10}$ In recent years, with increased use 
of immunotherapy, the incidence of irAEs is on the rise. It is widely recognized that for effective management of irAEs early recognition and treatment initiation are critical. However, in a survey conducted by the non-profit network Cancer Support Community, patients and caregivers reported difficulty in obtaining information about immunotherapy and identified information on managing side effects as their most important educational need. ${ }^{11}$ To address this need, several oncological societies, nonprofit organizations such as the American Cancer Society, and pharmaceutical companies have launched patient education programs, providing information in several formats, such as online and printed brochures, blogs, and testimonials. During clinic visits, patients and caregivers are now being provided information on the signs and symptoms that often herald the onset of impending irAEs. ${ }^{12}$ Regardless, patients' and caregivers' fear and anxiety, and feeling overwhelmed in their encounters with healthcare providers, may prevent their absorbing all of the provided information. ${ }^{13}$ Drug-specific wallet cards and educational apps may be used as part of the toolkit to reinforce the information provided and to remind patients and caregivers to watch for irAEs that can occur even after stopping treatment with immunotherapeutic agents. ${ }^{14}$ As irAEs are quite distinct from adverse effects of chemotherapeutic agents, this approach can also be used to inform the patients' local healthcare providers about toxic effects associated with immunotherapy and symptom monitoring requirements. Online social networks, such as Facebook, Twitter, Instagram, and YouTube, and support groups serve as platforms for patients and caregivers to provide and consume information on irAE management that can empower them to make informed decisions. ${ }^{15}$ However, privacy, confidentiality, and accuracy of the information shared is a concern.

Providing relevant and scientifically accurate information to patients even prior to initiating treatment allows patients to anticipate challenges and make informed decisions. ${ }^{10}$ However, engaging patients in their care is not without challenges. Although several delivery methods, such as audio or video recordings, educational classes, one-on-one educational sessions, and telephone calls, are available, retention of information is largely dependent on preferences and the emotional, literacy, and cultural needs of the patient. ${ }^{10} 16$ Furthermore, although early reporting of symptoms has been associated with improved treatment outcomes, including survival benefit, ${ }^{17}$ patients may be reluctant to report symptoms for fear of having to discontinue the anticancer therapy. Currently, there is no standard protocol on how to provide patient education that is tailored to the individual need of the patient. ${ }^{14}$ As a "one size fits all" approach to patient education may not produce the desired outcome, other need-based approaches, such as the $3 \mathrm{Ws}$ and an $\mathrm{H}$ process, in which four questions are discussed: (i) "who specifically is this resource for?"; (ii) "why should they read it?"; (iii) "what exactly do they want and need to know?"; and (iv) "how can the content best reach and engage the learner?,"13 may be considered to provide individualized patient education. The utilization of such approaches may provide an opportunity to develop effective and open communication channels between patients and healthcare providers that help to establish trust, set clear expectations, alleviate anxiety, and promote prompt reporting of symptoms.

\section{REFINING IRAE MANAGEMENT GUIDELINES}

Guidelines have been published by several key oncology societies (American Society of Clinical Oncology (ASCO), Society for Immunotherapy of Cancer (SITC), National Comprehensive Cancer Network, and European Society for Medical Oncology) to provide guidance on management of irAEs. ${ }^{4}{ }^{18-20}$ However, these guidelines are not evidence-based approaches, ${ }^{18}$ but rather algorithms, suggestions, and recommendations by panels of experts. ${ }^{4}$ In addition, although the expert panels that have developed these guidelines have been multidisciplinary, there has been a paucity of representation from key perspectives, such as emergency room physicians, anesthesiologists and surgeons, primary care physicians, patient advocates, and nurses. Inclusion of experts from these settings would add value. For example, as irAEs can lead to significant perioperative complications, such as electrolyte abnormalities and persistent hypotension due to adrenal insufficiency/ crisis secondary to immune-mediated hypopituitarism, inclusion of anesthesiologists on the panel would be helpful. ${ }^{21} 22$ Patient advocates can guide the expert panel to identify needs that are a priority to patients, help to assess risk-benefit ratios, provide insight on the impact of costs, and assess feasibility. ${ }^{23}$ Furthermore, patients may present with irAEs in any setting, such as urgent care or community clinics, where application of the current irAE management guidelines may be challenging due to lack of awareness and resources for coordinated patient care. ${ }^{24}$

To address these growing challenges, we recommend the following next steps: (i) convene an irAE Management Summit, possibly hosted by SITC with representation from all the afore-mentioned societies; (ii) develop toxicity-specific management committees with representation from relevant specialties that are tasked with reviewing and refining the current guidelines to create evidence-based expert consensus guidelines; (iii) publish the outcomes of the activities of the proposed summit; and (iv) make the summit a regularly planned effort incorporating the outcomes of new research. The development of consolidated and comprehensive irAE management guidelines that can be implemented not just at tertiary cancer care centers but also at any point of patient care could potentially optimize patient outcome. Of course, it is important to remember that use of such guidelines must be complemented by heightened awareness and informed clinical judgment. 


\section{STANDARDIZING REPORTING OF IRAES}

Typically, the Common Terminology Criteria for Adverse Events (CTCAE), ${ }^{25}$ which uses uniform terminology and a grading system to note severity, is used for standardized reporting of adverse events. However, there is growing evidence that the CTCAE do not capture all of the irAEs adequately, which may lead to misinterpretation of clinical observations. ${ }^{26}$ It is therefore necessary to add more terms to the CTCAE for standardized capture of all irAEs. To this end, the SITC CTCAE Task Force is now engaged in developing a module that includes irAEs, which could be incorporated into future versions of the CTCAE.

\section{OPTIMIZING THE CHOICE OF IMMUNOSUPPRESSIVE AGENTS}

Current guidelines ${ }^{48-20}$ rely heavily on the use of steroids for management of irAEs. The duration of steroid use and cumulative dose are usually much greater for management of irAEs compared with other clinical indications for corticosteroid use. ${ }^{27}$ However, long-term use of steroids gives rise to several concerns.

1 . Though the recommendation is to continue steroids until resolution of an irAE to grade 1 or better and subsequently taper the dose over a period of 4-6 weeks, efficacy of long-term steroid use in management of irAEs has not been validated. ${ }^{28}$ In contrast, when oral methylprednisolone was used as a 5-day tapered dose pack (steroid burst) for mild irAEs, all patients had recurrent grade 2 irAEs. ${ }^{28}$ Thus, the optimal dose and duration of steroid use with minimal risk of side effects in the management of irAEs are unknown.

2. Long-term steroid use may be associated with other complications, such as gastritis, hypertension, newonset hyperglycemia, and opportunistic infections (eg, pneumocystis pneumonia). ${ }^{18}$

Hence, there is a growing interest in managing irAEs using steroid-sparing immune-modulating agents. Several biological immunosuppressive agents such as antitumor necrosis factor- $\alpha$ antibody infliximab, gut-specific immunosuppressant vedolizumab, antithymocyte globulin, antiCD20 monoclonal antibody rituximab, and non-biological immunosuppressive agents such as mycophenolate mofetil, calcineurin inhibitors, intravenous immunoglobulin, cyclophosphamide, and methotrexate have been used in the management of severe or steroid-refractory irAEs. ${ }^{18} 20$ 29-33 There have been efforts, including clinical trials, to develop therapeutics that target the unique pathways or factors driving the specific toxicities. In some lifethreatening steroid-refractory irAEs, cytokine-directed monoclonal antibodies such as tocilizumab (an interleukin 6 (IL-6) receptor antagonist) have been used initially to shut down a rapidly evolving immunological process that would lead to release of cytokines by hyperactivated T cells. ${ }^{34}$ Emerging data suggest that Th17-associated cytokines, particularly IL-23 and IL-17, contribute to pathogenesis of immune-mediated colitis and dermatitis. Thus, therapeutic agents targeting IL-17 (ixekizumab, brodalumab, and secukinumab) are potentially available for use in management of severe psoriasis refractory to anti-TNF $\alpha$ therapy and rheumatoid arthritis and anti-IL-6 refractory irAEs, ${ }^{32}$ and an agent targeting IL-23 (ustekinumab) could be used for anti-TNF $\alpha$-refractory irAEs. However, these agents' use in the management of irAEs may be limited based on their mechanism of action. ${ }^{33}$ For example, while infliximab is contraindicated in hepatic irAEs and gastrointestinal perforation, mycophenolate mofetil is efficacious in ICPi-induced hepatitis and steroid-refractory colitis. Another strategy that holds promise is targeting of signaling pathways; aberrant JAK-STAT pathway and mTOR pathway signaling have been implicated in autoimmunity and cancer. ${ }^{35}$ Because the use of these immune-modulating agents has been extrapolated based on their application in autoimmune conditions, their safety and efficacy in irAE management and their impact on response to ICPi therapy should be prospectively evaluated in clinical trials.

Furthermore, there exists uncertainty regarding the impact of chronic immunosuppression on the efficacy of ICPis in treatment of cancer in patients with pre-existing autoimmune diseases and in transplant recipients. Data suggest that patients receiving immunosuppressants for pre-existing conditions had fewer irAEs than those who were not; however, cancer control was better in patients who had irAEs. ${ }^{36}$ Therefore, prospective studies to optimize the choice, dosing, and duration of immunosuppressants for prevention of autoimmune flare and graft rejection and management of irAEs are needed. The primary aims of such trials should be to (i) shorten the duration of irAEs with sparing of the affected organ system, (ii) enable possible rechallenge with ICPis after resolution of irAE symptoms, and (iii) not dampen the immune response elicited by ICPis.

\section{PURSUING BETTER UNDERSTANDING OF IRAES}

As the mechanism underlying the toxic effects of ICPis is not fully defined, several key questions remain unanswered. Some of them are:

1. After adjustment for factors such as class of the drug, tumor type, age, race, and sex, why do patients have the same irAE but different severities, different irAEs of the same severity, or different irAEs of different severities?

2. What makes some patients more susceptible to irAEs, and what makes some organs more susceptible to irAEs?

3. As mechanisms underlying irAEs are thought to be driven by autoimmunity, does germline genetic variation affect risk of irAEs? Prospective biomarker-driven multicentric studies must be implemented to investigate germline predisposition to irAEs, tumor-specific irAEs, treatment-specific irAEs, and organ-specific irAEs.

4. Will characterization of immune-effector pathways driving irAEs inform the choice of immunomodulatory agents used in management of irAEs? 
5. Is it possible that in some cases the toxic effect results from the immune system's attacking what most resembles the tumor due to shared expression of antigens between tumor and normal cells (eg, vitiligo in patients with melanoma)?

6. What is the relationship between irAEs and response to ICPis? Although there are some reports of association between irAEs and improved treatment outcomes, ${ }^{9}{ }^{37-40}$ only certain irAEs are associated with improved response rates or overall survival. Therefore, it is important to understand the mechanisms that explain such associations at the molecular level. Though several analyzes, such as $\mathrm{T}$ cell receptor clonotype analysis, ${ }^{41}$ thyroid studies, ${ }^{42}$ and microbiome studies, ${ }^{43}$ have been performed, the link between irAEs and response has not yet been elucidated.

7. What are the risk and effectiveness of vaccines in patients on immunotherapy-based treatment, particularly in the face of the COVID-19 pandemic?

8. What is the role of antiviral, antibacterial, or antifungal prophylaxis, as there are several concerns about the risk of infections in patients with cancer treated with immunotherapy-based regimens? ICPis are increasingly used in combination with cytotoxic chemotherapies that are known to predispose patients to life-threatening infections due to drug-induced myelosuppression. However, retrospective studies in patients with cancer receiving ICPis in combination with chemotherapy showed that although neutropenia is an independent risk factor for infection, the infection rate in patients receiving combination therapy was comparable to the rate in patients receiving chemotherapy alone. ${ }^{44}$ Use of ICPis has been associated with increased risk of serious infection, ${ }^{45}$ particularly opportunistic infections such as invasive aspergillosis, cytomegalovirus-induced hepatitis, and pneumocystis pneumonia. Rarely, immune modulation may unmask chronic underlying infections such as mycobacterium tuberculosis. ${ }^{46}$ In recent days, ICPi use within 90 days has been identified as the single highest risk factor for hospitalization and severe COVID-19. ${ }^{47}$ As data continues to evolve, validation of the finding in larger studies is needed.

As our endeavor to understand irAEs continue, more (i) preclinical, (ii) translational, and (iii) clinical studies are needed to fill these knowledge gaps.

\section{INCLUDING HIGH-RISK PATIENTS}

Special population groups have been excluded from enrollment on immunotherapy-based clinical trials due to increased risk for irAEs, exacerbations of autoimmunity, or viral reactivation. ${ }^{48}$ These populations include patients with a history of primary or secondary immune deficiencies, autoimmune diseases, stem cell or solid organ transplantation (SOT), HIV, hepatitis B or C, or prior irAEs. However, such patients have an increased risk of developing cancer, so their inclusion in immunotherapybased trials is important.

SOT recipients in particular have increased cancer risk as they are subject to chronic immunosuppression to prevent transplant rejection. Although use of ICPis is limited in SOT recipients, rapid allograft rejection and high mortality rates secondary to graft rejection have been reported soon after initiation of treatment with ICPis. ${ }^{490}$ The rejection rate was higher in those receiving low-dose prednisone than in those receiving other immunosuppressive therapies. ${ }^{49}$ Similarly, in patients with relapsed classical Hodgkin's lymphoma, although response rates as high as $75 \%-95 \%$ were observed with ICPis for relapse after allogeneic hematopoietic cell transplantation, graftversus-host disease was reported in $30 \%$ of the cases and was associated with a high mortality rate.$^{51}{ }^{52}$ Clinical trials are currently enrolling SOT recipients with cancer to evaluate safety and efficacy of ICPis in this patient population (NCT04339062, NCT03816332).

On the contrary, favorable outcome has been reported in HIV-positive patients with cancer treated with ICPis. The use of ICPis in this population is supported by the increased PD-L1 expression and cytotoxic T cell and activated macrophage infiltration in tumor samples of HIVpositive patients with non-small cell lung cancer ${ }^{53}$ and better viral control with PD-1/PD-L2 blockade. ${ }^{54}$ Limited data from retrospective studies suggest that the efficacy of ICPis in this patient population is comparable to that in the general population. ${ }^{36545}$ Until recently, patients with cancer with HIV were excluded from most immunotherapybased clinical trials. ${ }^{56}$ However, the National Cancer Institute and the Food and Drug Administration (FDA) have encouraged inclusion of patients with cancer with HIV, hepatitis B, or hepatitis C in cancer clinical trials ${ }^{57}$ (NCT03603808, NCT02595866, NCT02408861). While there may be some level of justification in not including such patient populations for first-in-human and first-inclass early phase immunotherapy-based clinical trials, it is reasonable to include them in mature-phase trials after safety of the drug has been reasonably established. Recently, prospective studies of nivolumab and pembrolizumab in patients with HIV infection have reported an acceptable safety profile with tumor responses in nonsmall cell lung cancer, Kaposi sarcoma, non-Hodgkin's lymphoma, and hepatocellular carcinoma. ${ }^{58} 59$

Similarly, data from a few case reports and retrospective studies conducted in patients with melanoma and non-small cell lung cancer with autoimmune conditions suggest that although treatment-emergent deaths have been reported in a few patients, autoimmune disease flares and irAEs are manageable in most patients with preexisting autoimmune diseases and do not always require termination of ICPi therapy ${ }^{3660}$ In these reports, patients who received immunosuppressants at the start of ICPi therapy had fewer adverse events compared with those who did not. However, patients who had adverse events had higher response rates compared with those who did not have adverse events, suggesting that occurrence 
of irAE or autoimmune flare may be associated with response. ${ }^{36}$ Studies show that PD-1 inhibitors have significant antitumor activity at dose levels lower than those currently approved by FDA, ${ }^{61}$ suggesting that ICPis at lower dose levels may be used as a strategy to mitigate risk of irAEs without negating antitumor activity. These observations are at best hypothesis-generating. Currently, an early phase clinical trial (NCT03816345) evaluating nivolumab in patients with cancer with autoimmune disease is under way.

As most studies that comprised high-risk patients have not included immune markers, factors associated with response or toxic effects from ICPis in patients with immune deficiencies, autoimmune diseases, prior transplantation, HIV, hepatitis B or C, or prior irAEs are unknown. In the absence of an evidence-based rationale for withholding use of ICPis in such special populations, more patients with cancer in this high-risk group are likely to use ICPis in the real-world setting following their regulatory approval for treatment of various cancers. Nevertheless, as maintaining a balance between treating cancer and preventing autoimmune flare or graft rejection or viral reactivation is difficult, this approach has to be used with caution and after well-informed discussion with the patient and caregivers. Currently, there are no guidelines for the treatment of patients with ICPis in the setting of a prior organ transplant or autoimmune disease. However, autoimmune flare and graft rejection were more common with PD-1 inhibitors than with CTLA-4 inhibitors. Furthermore, as it is perceived that immunosuppression prior to initiation of ICPi therapy could blunt the ability of the ICPi to evoke an immune response, ${ }^{62}$ it is important to answer questions such as how to taper down immunosuppressants before initiating ICPi therapy and how to ramp them back up as irAEs occur. Therefore, carefully designed prospective studies to identify optimal anti-cancer therapies, dosing strategies, class and dose of immunosuppressant, and time since transplant are needed. Due to the rarity of use of ICPis in this patient population, a national registry can be established to enroll these patients for future studies. Developing such clinical trials with close monitoring strategies and translational studies will advance our understanding of riskbenefit ratios and underlying mechanisms of response and irAEs in this high-risk population.

\section{INCORPORATING DIAGNOSTIC TOOLS TO PERSONALIZE IRAE MANAGEMENT}

It is widely recognized that effective management of irAEs is dependent on early recognition and prompt intervention. Though several markers-such as absolute lymphocyte, eosinophil, and neutrophil counts ${ }^{63}$; T cell repertoire; pre-existing autoantibodies and singlenucleotide polymorphisms ${ }^{64}$; and circulating biomarkers during treatment such as miRNAs, cytokines, and chemokines $^{65}$ - have been investigated, along with the microbiome, ${ }^{66}$ no validated biomarkers are currently available to predict patients at risk of developing irAEs or to personalize a monitoring plan to detect emergence of irAEs. Novel diagnostic tools to predict patients' baseline risk for developing irAEs, such as radiomics for pneumonitis ${ }^{67}$ and $\mathrm{T}$ cell receptor beta variable region sequencing for severe irAEs, ${ }^{68} 69$ should be validated in large clinical trials for reliability and generalizability. As these tools use routinely collected blood samples and CT images, if validated, they could be readily available in the clinic to facilitate decision making.

\section{USING WIRELESS TECHNOLOGY AND DIGITAL HEALTH}

As time is of the essence in managing irAEs efficiently, advances in wireless technology and emerging digital modalities can be used to streamline communication and empower healthcare providers to make informed decisions. ${ }^{71}$ Partnerships between technology teams, industry, professional organizations, and academic centers can lead to development of inexpensive medical apps with the objective of providing high-quality care even in remote locations. Recent launching of the IO Tox Management app (The Ronin Project, LLC, V.1) ${ }^{72}$ to equip healthcare providers with a resource to manage irAEs based on guidelines from the ASCO and toxicity grading from the National Cancer Institute marks significant progress in this area. Importantly, the timeliness and availability of ready-to-use resources are invaluable both to patients and healthcare providers, especially with current mobility restrictions due to the COVID-19 pandemic.

Furthermore, information collected beyond clinical visits through wireless technology, such as patientreported outcomes (PROs), can be used to monitor symptoms for emergence of irAEs. As prompt interventions based on collected information have been shown to improve survival outcomes in patients with cancer, ${ }^{17} \mathrm{PROs}$ can also be used to detect emerging irAEs, enabling initiation of appropriate monitoring and irAE management measures. Recently, a smartphone-based app was used to monitor diarrhea and hypertension in patients with ovarian cancer receiving the combination of olaparib and cediranib. ${ }^{73}$ Self-reporting of symptoms using this app allowed healthcare providers to analyze patient data in real time and triage management of acute treatmentrelated adverse events. Such applications using wearables and sensors could be expanded to monitor patients treated with immunotherapy for emergence of irAEs, as finding irAEs early and holding therapy, not adding more fuel to the fire, can be helpful in keeping the immune activation at a level at which it recognizes the tumor but not the host.

\section{PROVIDING A PLATFORM TO HEAR THE MISSING PATIENT'S VOICE}

Data on toxicities associated with ICPis are generally collected via tabulation of irAEs, which are graded by clinicians. However, it is well known that clinicians typically 
underestimate the symptoms of toxicity experienced by patients. ${ }^{745}$ PROs provide a platform by which clinicians hear the missing patient voice on the debilitating side effects of treatment and their impact on functioning. Patients' self-reports of their symptoms before and during treatment are needed because changes in symptoms often serve as an early indicator of either treatment benefit or toxicity ${ }^{12}$ or survival outcome. ${ }^{76}$ This information is vital for clinicians because it determines patients' ability to tolerate the intended oncological therapies and allows for improved patient-centered care. ${ }^{77} 78$ For example, early and effective supportive care intervention may improve a patient's tolerance of the drug, increase adherence to treatments, enhance health-related quality of life, and ultimately improve survival outcomes. ${ }^{76}$ As assessing symptoms between visits allows better understanding of the symptomatic adverse effects of treatment as well as better tracking of disease-related symptoms, ${ }^{80} 81$ a validated symptom assessment tool such as the MD Anderson Symptom Inventory, ${ }^{82}$ which can be completed at home, may be used to assess longitudinal changes in symptoms.

\section{SHARING EVOLVING DATA}

Data sharing is integral to rapid advancement of the field of medicine. Data sharing in biomedical research can promote easy access to knowledge that has been painstakingly acquired and prevent duplication of research activities. ${ }^{83}$ Dissemination of the results of clinical trials, including information on irAEs, management of irAEs, and outcomes of such management, in a timely manner will not only enhance our understanding of irAEs but also expedite the translation of research results into knowledge, products, and procedures to improve human health through development of potentially life-saving strategies to mitigate irAEs.

In conclusion, in recent years we have witnessed a dramatic improvement in the treatment of different types of cancer with the introduction of ICPis. Even as the field of immune-oncology is rapidly evolving, we have made significant strides in understanding and managing irAEs effectively. However, there is room for further improvement. By adopting strategies such as listening to patients' voices, conducting preclinical, clinical, and translational studies to identify predictive markers of irAEs, and developing evidence-based irAE management guidelines, we can minimize the toxic effects of ICPis while achieving the full therapeutic potential of these drugs.

\section{Twitter Aung Naing @AnaingMD and James L Gulley @gulleyj1}

Acknowledgements We thank Bettzy Stephen, Department of Investigational Cancer Therapeutics at MD Anderson, and Sunita C Patterson and Bryan F Tutt, Research Medical Library at MD Anderson for providing editorial assistance.

Contributors AN wrote the initial draft. All authors provided critical feedback for intellectual content of manuscript and approved the final version of the manuscript for publication.

Funding The authors have not declared a specific grant for this research from any funding agency in the public, commercial or not-for-profit sectors.
Competing interests None declared.

\section{Patient consent for publication Not required.}

Provenance and peer review Not commissioned; externally peer reviewed.

Open access This is an open access article distributed in accordance with the Creative Commons Attribution Non Commercial (CC BY-NC 4.0) license, which permits others to distribute, remix, adapt, build upon this work non-commercially, and license their derivative works on different terms, provided the original work is properly cited, appropriate credit is given, any changes made indicated, and the use is non-commercial. See http://creativecommons.org/licenses/by-nc/4.0/.

\section{ORCID iDs}

Aung Naing http://orcid.org/0000-0002-4803-8513

Joud Hajjar http://orcid.org/0000-0003-4633-1043

James L Gulley http://orcid.org/0000-0002-6569-2912

Michael B Atkins http://orcid.org/0000-0003-3901-9924

Patrick Hwu http://orcid.org/0000-0003-0554-2856

\section{REFERENCES}

1 Martins F, Sofiya L, Sykiotis GP, et al. Adverse effects of immunecheckpoint inhibitors: epidemiology, management and surveillance. Nat Rev Clin Oncol 2019;16:563-80.

2 Postow MA, Sidlow R, Hellmann MD. Immune-related adverse events associated with immune checkpoint blockade. N Engl J Med 2018;378:158-68.

3 Wang Y, Zhou S, Yang F, et al. Treatment-related adverse events of PD-1 and PD-L1 inhibitors in clinical trials: a systematic review and meta-analysis. JAMA Oncol 2019;5:1008-19.

4 Brahmer JR, Lacchetti C, Schneider BJ, et al. Management of immune-related adverse events in patients treated with immune checkpoint inhibitor therapy: American Society of clinical oncology clinical practice guideline. J Clin Oncol 2018;36:1714-68.

5 Ramos-Casals M, Brahmer JR, Callahan MK, et al. Immune-related adverse events of checkpoint inhibitors. Nat Rev Dis Primers 2020;6:38.

6 van Holstein Y, Kapiteijn E, Bastiaannet E, et al. Efficacy and adverse events of immunotherapy with checkpoint inhibitors in older patients with cancer. Drugs Aging 2019;36:927-38.

7 Balaji A, Zhang J, Wills B, et al. Immune-related adverse events requiring hospitalization: spectrum of toxicity, treatment, and outcomes. J Oncol Pract 2019;15:e825-34.

8 Wang DY, Salem J-E, Cohen JV, et al. Fatal toxic effects associated with immune checkpoint inhibitors: a systematic review and metaanalysis. JAMA Oncol 2018;4:1721-8.

9 Teulings H-E, Limpens J, Jansen SN, et al. Vitiligo-like depigmentation in patients with stage III-IV melanoma receiving immunotherapy and its association with survival: a systematic review and meta-analysis. J Clin Oncol 2015;33:773-81.

10 Adams RJ. Improving health outcomes with better patient understanding and education. Risk Manag Healthc Policy 2010;3:61-72.

11 Aspiras A, Power NJ, Gonzalo MB. Survey analysis: assessing the needs of immunotherapy patients, caregivers, and health care providers. JCO 2018;36:135.

12 Mendoza TR, Zhao F, Cleeland CS, et al. The validity and utility of the M. D. anderson symptom inventory in patients with breast cancer: evidence from the symptom outcomes and practice patterns data from the eastern cooperative Oncology Group. Clin Breast Cancer 2013;13:325-34.

13 Papadakos CT, Papadakos J, Catton P, et al. From theory to pamphlet: the $3 \mathrm{Ws}$ and an $\mathrm{H}$ process for the development of meaningful patient education resources. J Cancer Educ 2014;29:304-10.

14 Kinnaer L-M, De Coster S, Coolbrandt A, et al. Key elements for the education and counselling of patients treated with oral anticancer drugs. Eur J Oncol Nurs 2019;41:173-94.

15 De Martino I, D'Apolito R, McLawhorn AS, et al. Social media for patients: benefits and drawbacks. Curr Rev Musculoskelet Med 2017;10:141-5.

16 Valenti RB. Chemotherapy education for patients with cancer: a literature review. Clin J Oncol Nurs 2014;18:637-40.

17 Basch E, Deal AM, Dueck AC, et al. Overall survival results of a trial assessing patient-reported outcomes for symptom monitoring during routine cancer treatment. JAMA 2017;318:197-8.

18 Puzanov I, Diab A, Abdallah K, et al. Managing toxicities associated with immune checkpoint inhibitors: consensus recommendations 
from the Society for immunotherapy of cancer (SITC) toxicity management Working group. J Immunother Cancer 2017;5:95.

19 Haanen JBAG, Carbonnel F, Robert C, et al. Management of toxicities from immunotherapy: ESMO clinical practice guidelines for diagnosis, treatment and follow-up. Ann Oncol 2017;28:iv119-42.

20 Thompson JA, Schneider BJ, Brahmer J, et al. Management of Immunotherapy-Related toxicities, version 1.2019. J Natl Compr Canc Netw 2019;17:255-89.

21 Norman PH, Calderon TM, Wang Y, et al. Inclusion of anesthesiologists and surgeons while developing guidelines for management of immune-related adverse events. $J$ Immunother Precis Oncol 2020;3:56-7.

22 Lewis AL, Chaft J, Girotra M, et al. Immune checkpoint inhibitors: a narrative review of considerations for the anaesthesiologist. $\mathrm{Br} J$ Anaesth 2020;124:251-60.

23 Alonso-Coello P, Oxman AD, Moberg J, et al. Grade evidence to decision (ETD) frameworks: a systematic and transparent approach to making well informed healthcare choices. 2: clinical practice guidelines. BMJ 2016;353:i2089.

24 Cole S, Zibelman M, Bertino E, et al. Managing immuno-oncology toxicity: top 10 innovative institutional solutions. Am Soc Clin Oncol Educ Book 2019;39:96-104.

25 U.S. Department of Health and Human Services. Common terminology criteria for adverse events (CTCAE) version 5.0. Services USDoHaH, 2017

26 Yu Y, Ruddy KJ, Tsuji S, et al. Coverage evaluation of CTCAE for capturing the immune-related adverse events Leveraging text mining technologies. AMIA Jt Summits Trans/ Sci Proc 2019;2019:771-8.

27 Agarwal K, Yousaf N, Morganstein D. Glucocorticoid use and complications following immune checkpoint inhibitor use in melanoma. Clin Med 2020;20:163-8.

28 Williams KJ, Grauer DW, Henry DW, et al. Corticosteroids for the management of immune-related adverse events in patients receiving checkpoint inhibitors. J Oncol Pharm Pract 2019;25:544-50.

29 Bergqvist V, Hertervig E, Gedeon P, et al. Vedolizumab treatment for immune checkpoint inhibitor-induced enterocolitis. Cancer Immunol Immunother 2017;66:581-92.

30 Abu-Sbeih H, Ali FS, Alsaadi D, et al. Outcomes of vedolizumab therapy in patients with immune checkpoint inhibitor-induced colitis: a multi-center study. J Immunother Cancer 2018;6:142.

31 Chmiel KD, Suan D, Liddle C, et al. Resolution of severe ipilimumabinduced hepatitis after antithymocyte globulin therapy. $J$ Clin Oncol 2011;29:e237-40.

32 Martins F, Sykiotis GP, Maillard M, et al. New therapeutic perspectives to manage refractory immune checkpoint-related toxicities. Lancet Oncol 2019;20:e54-64.

33 Wang A, Xu Y, Fei Y, et al. The role of immunosuppressive agents in the management of severe and refractory immune-related adverse events. Asia Pac J Clin Oncol 2020;16:201-10.

34 Stroud CR, Hegde A, Cherry C, et al. Tocilizumab for the management of immune mediated adverse events secondary to PD-1 blockade. J Oncol Pharm Pract 2019;25:551-7.

35 Esfahani K, Elkrief A, Calabrese C, et al. Moving towards personalized treatments of immune-related adverse events. Nat Rev Clin Oncol 2020;17:504-15.

36 Abdel-Wahab N, Shah M, Lopez-Olivo MA, et al. Use of immune checkpoint inhibitors in the treatment of patients with cancer and preexisting autoimmune disease. Ann Intern Med 2018;169:133-4.

37 Fujii T, Colen RR, Bilen MA, et al. Incidence of immune-related adverse events and its association with treatment outcomes: the MD anderson cancer center experience. Invest New Drugs 2018;36:638-46.

38 Attia P, Phan GQ, Maker AV, et al. Autoimmunity correlates with tumor regression in patients with metastatic melanoma treated with anti-cytotoxic T-lymphocyte antigen-4. J Clin Oncol 2005:23:6043-53.

39 Freeman-Keller M, Kim Y, Cronin $\mathrm{H}$, et al. Nivolumab in resected and unresectable metastatic melanoma: characteristics of immunerelated adverse events and association with outcomes. Clin Cancer Res 2016;22:886-94

$40 \mathrm{Kim} \mathrm{HI}$, Kim M, Lee S-H, et al. Development of thyroid dysfunction is associated with clinical response to PD-1 blockade treatment in patients with advanced non-small cell lung cancer. Oncoimmunology 2018; $7:$ e1375642

41 Berner F, Bomze D, Diem S, et al. Association of checkpoint inhibitorinduced toxic effects with shared cancer and tissue antigens in nonsmall cell lung cancer. JAMA Oncol 2019;5:1043-7.

42 Atkins MB, Mier JW, Parkinson DR, et al. Hypothyroidism after treatment with interleukin-2 and lymphokine-activated killer cells. $N$ Engl J Med 1988;318:1557-63.
43 Chaput N, Lepage P, Coutzac C, et al. Baseline gut microbiota predicts clinical response and colitis in metastatic melanoma patients treated with ipilimumab. Ann Oncol 2019;30:30

44 Malek A, Khalil M, Hachem R, et al. Impact of checkpoint inhibitor immunotherapy primarily pembrolizumab on infection risk in patients with advanced lung cancer: a comparative retrospective cohort study. Clin Infect Dis 2020. doi:10.1093/cid/ciaa802. [Epub ahead of print: 21 Jun 2020]

45 Del Castillo M, Romero FA, Argüello E, et al. The spectrum of serious infections among patients receiving immune checkpoint blockade for the treatment of melanoma. Clin Infect Dis 2016;63:1490-3.

46 Anastasopoulou A, Ziogas DC, Samarkos M, et al. Reactivation of tuberculosis in cancer patients following administration of immune checkpoint inhibitors: current evidence and clinical practice recommendations. J Immunother Cancer 2019;7:239.

47 Robilotti EV, Babady NE, Mead PA, et al. Determinants of COVID-19 disease severity in patients with cancer. Nat Med 2020;26:1218-23.

48 Hajjar J. Cancer immunotherapy for the immunosuppressed: dissecting the conundrum of safety and efficacy. $J$ Immunother Precis Oncol 2019;2:53-4.

49 Abdel-Wahab N, Safa H, Abudayyeh A, et al. Checkpoint inhibitor therapy for cancer in solid organ transplantation recipients: an institutional experience and a systematic review of the literature. $J$ Immunother Cancer 2019;7:106

50 Fisher J, Zeitouni N, Fan W, et al. Immune checkpoint inhibitor therapy in solid organ transplant recipients: a patient-centered systematic review. J Am Acad Dermatol 2020;82:1490-500.

51 Haverkos BM, Abbott D, Hamadani M, et al. Pd-1 blockade for relapsed lymphoma post-allogeneic hematopoietic cell transplant: high response rate but frequent GVHD. Blood 2017;130:221-8.

52 Herbaux C, Gauthier J, Brice P, et al. Efficacy and tolerability of nivolumab after allogeneic transplantation for relapsed Hodgkin lymphoma. Blood 2017:129:2471-8.

53 Domblides C, Antoine M, Hamard C, et al. Nonsmall cell lung cancer from HIV-infected patients expressed programmed cell death-ligand 1 with marked inflammatory infiltrates. AIDS 2018;32:461-8.

54 Cook MR, Kim C. Safety and efficacy of immune checkpoint inhibitor therapy in patients with HIV infection and advanced-stage cancer: a systematic review. JAMA Oncol 2019;5:1049-54.

55 Granwehr B. Review: the impact of HIV infection on cancer treatment with immunotherapy. J Immunother Precis Oncol 2019;2:85-92.

56 Vora KB, Awad MM. Exclusion rates of patients living with HIV from cancer immunotherapy clinical trials. Journal of Clinical Oncology 2020;38:e19035.

57 Uldrick TS, Ison G, Rudek MA, et al. Modernizing clinical trial eligibility criteria: recommendations of the American Society of clinical Oncology-Friends of cancer research HIV Working group. $J$ Clin Oncol 2017;35:3774-80.

58 Lavolé A, Guihot A, Veyri M, et al. Pd-1 blockade in HIV-infected patients with lung cancer: a new challenge or already a strategy? Ann Oncol 2018;29:1065-6.

59 Uldrick TS, Gonçalves PH, Abdul-Hay M, et al. Assessment of the safety of pembrolizumab in patients with HIV and advanced cancer-a phase 1 study. JAMA Oncol 2019. doi:10.1001/ jamaoncol.2019.2244. [Epub ahead of print: 02 Jun 2019]

60 Boland P, Pavlick AC, Weber J, et al. Immunotherapy to treat malignancy in patients with pre-existing autoimmunity. J Immunother Cancer 2020;8:e000356.

61 Renner A, Burotto M, Rojas C. Immune checkpoint inhibitor dosing: can we go lower without compromising clinical efficacy? J Glob Oncol 2019;5:1-5.

62 Kittai AS, Oldham H, Cetnar J, et al. Immune checkpoint inhibitors in organ transplant patients. J Immunother 2017;40:1-81.

63 Huebener P, Pradere J-P, Hernandez C, et al. The HMGB1/RAGE axis triggers neutrophil-mediated injury amplification following necrosis. $J$ Clin Invest 2015;125:539-50.

64 June $\mathrm{CH}$, Warshauer JT, Bluestone JA. Is autoimmunity the Achilles' heel of cancer immunotherapy? Nat Med 2017;23:540-7.

65 Tarhini AA, Zahoor H, Lin Y, et al. Baseline circulating IL-17 predicts toxicity while TGF- $\beta 1$ and IL-10 are prognostic of relapse in ipilimumab neoadjuvant therapy of melanoma. J Immunother Cancer 2015;3:39.

66 Chaput N, Lepage P, Coutzac C, et al. Baseline gut microbiota predicts clinical response and colitis in metastatic melanoma patients treated with ipilimumab. Ann Oncol 2017;28:1368-79.

67 Colen RR, Fujii T, Bilen MA, et al. Radiomics to predict immunotherapy-induced pneumonitis: proof of concept. Invest New Drugs 2018;36:601-7.

68 Looney TJ, Duose DY, Lowman G, et al. Haplotype analysis of the T-cell receptor beta (TCRB) locus by long-amplicon TCRB repertoire sequencing. J Immunother Precis Oncol 2019:137-43. 
69 Looney T, Linch E, Lowman G, et al. Evaluating the link between T cell receptor beta variable gene polymorphism and immune mediated adverse events during checkpoint blockade immunotherapy. JCO 2018;36:e15002-e.

70 Ventola CL. Mobile devices and apps for health care professionals: uses and benefits. P T 2014;39:356-64

71 Dicker AP, Jim HSL. Intersection of digital health and oncology. JCO Clin Cancer Inform 2018;2:1-4.

72 IO Tox Management. The Ronin project I.Version 1.0.3, 2020. Available: https://apps.apple.com/us/app/io-tox-management/ id1514006592 [Accessed 14 Aug 2020].

73 Liu JF, Lee J-M, Strock E, et al. Technology applications: use of digital health technology to enable drug development. JCO Clin Cancer Inform 2018;2:1-12.

74 Basch E, Bennett A, Pietanza MC. Use of patient-reported outcomes to improve the predictive accuracy of clinician-reported adverse events. J Natl Cancer Inst 2011;103:1808-10.

75 Basch $\mathrm{E}$. The missing voice of patients in drug-safety reporting. $N$ Engl J Med 2010;362:865-9.

76 Wang XS, Shi Q, Lu C, et al. Prognostic value of symptom burden for overall survival in patients receiving chemotherapy for advanced nonsmall cell lung cancer. Cancer 2010;116:137-45.

77 Basch E, lasonos A, McDonough T, et al. Patient versus clinician symptom reporting using the National cancer Institute common terminology criteria for adverse events: results of a questionnairebased study. Lancet Oncol 2006;7:903-9.

78 Kluetz PG, Chingos DT, Basch EM, et al. Patient-reported outcomes in cancer clinical trials: measuring symptomatic adverse events with the National cancer Institute's patient-reported outcomes version of the common terminology criteria for adverse events (PRO-CTCAE). Am Soc Clin Oncol Educ Book 2016;35:67-73.

79 Cleeland CS, Sloan JA, Cella D, et al. Recommendations for including multiple symptoms as endpoints in cancer clinical trials. Cancer 2013:119:411-20.

80 Schadendorf D, Dummer R, Hauschild A, et al. Health-related quality of life in the randomised KEYNOTE-002 study of pembrolizumab versus chemotherapy in patients with ipilimumab-refractory melanoma. Eur J Cancer 2016;67:46-54.

81 Mendoza T. Understanding the toxicity of cancer immunotherapies: use of patient-reported outcomes. J Immunother Precis Oncol 2018;1:38-45.

82 Mendoza T, Sheshadri A, Altan M, et al. Evaluating the psychometric properties of the immunotherapy module of the MD anderson symptom inventory. J Immunother Cancer 2020;8:e000931.

83 Rutella S, Cannarile MA, Gnjatic S, et al. Society for immunotherapy of cancer clinical and biomarkers data sharing resource document: volume I-conceptual challenges. J Immunother Cancer 2020;8:e001389. 